\title{
The Fundament of the Mass and Effects of the Gravitation on a Particle and Light in the Mass, Time, Distance, Velocity, Frequency, Wavelength: Variant Mass for a Particle which emits Gravitational Energy for a Particle Orbiting a large Planet or Sun and for a Binary Star and Variant Frequency for the Light Passing Close a Gravitational Field from a Massive Object (Sun)
}

\section{Giovanni Alcocer}

Independent Research, Guayaquil Ecuador. Email: giov_alc_science@hotmail.com

Master in Physics with specialization in Astrophysics and Medical Physics, Professor of Physics, Advanced Mathematics and Science in general.

\author{
DOI:10.46382/MJBAS.2020.4305
}

Albert Einstein wrote in a research article: "Does the inertia of a body depend on its energy content?” (Ist die Trägheit eines Körpers von seimen Energienhalt abhängig?) [1]: "If a body emits energy $E$ in the form of radiation, its mass decreases by E/c ${ }^{2}$. This is true for any type of radiation emitted (gravitational or electromagnetic energy) which produce a decrease in the mass of the body. Thus, Maxwell's theory shows that electromagnetic waves are radiated whenever charges accelerate as for example for the electrón. Then, this electromagnetic radiation (photons) produces decreases in the mass of the electron which is given by the formula of the Variant Mass for an Accelerated Charged Particle which was demonstrated by myself at this research [2]. The objective of this research is to demonstrate by theory, experiment and result the discovery formula which describe exactly the variant mass of a particle which emits gravitational energy. The results of the mass formula are of great relevance for Gravitational Interactions. It is in accordance with the classic result for the emission of the total gravitational energy (bond total energy) for a particle orbiting a large Planet or Sun and for a Binary Star. It is also demonstrated and explained the effects of the gravitation in a particle or light and the Perihelion Precession of Mercury. The formula for the gravitation redshift frequency, the wavelength, the light velocity, time measurement and the decreasing radius for a particle in a gravitational field are demonstrated. The formula of the light velocity is tested for the deflection of light passing close to the sun. The formula for time dilation and decrease distance are used to calculate the Perihelion Precession of Mercury. It is in agreement with the experiment result and with the Theory of General Relativity. The consequences of this research are amazing and in accordance with the same General Theory of Relativity, Newton Theory and with profound Insignia in Quantum Mechanics and for the Unification Theory.

Keywords: Fundament of mass, Variant mass, Gravitational energy, Variant frequency, Gravitational redshift, Total bond energy, Binary stars.

\section{Introduction}

Albert Einstein wrote in an research article entitled: "Does the inertia of a body depend on its energy content? [1]". Thus, if a particle emits radiation energy $\mathrm{E}$ (electromagnetic or gravitational), then the rest energy of the particle decreases in $\mathrm{E}$ and by the mass-energy conservation also the mass of the decreases at the same rate $\mathrm{E} / \mathrm{c}^{2}$ [2]. The mass of a body is a measure of its energy content. The inert mass of a body increase or decrease with energy that it contains. If the initial mass of the particle is $\mathbf{M}$ and th final mass of the particle is $\mathbf{M}^{\prime}$, then it is obtained: $\mathrm{M}^{\prime} \mathrm{M}^{\prime}=\mathrm{E} / \mathrm{c}^{2}, \mathrm{E}=\left(\mathrm{M}^{\prime}-\mathrm{M}^{\prime}\right) \mathrm{c}^{2} \& \mathrm{E}=\Delta \mathrm{Mc}^{2}$

The decrease or increase in mass $\Delta \mathrm{M}=\mathrm{M}-\mathrm{M}^{\prime}$ of the particle is linked to the radiation energy $\mathrm{E}$ emitted or absorbed. This energy is also the lost energy of the particle. The radiation carries inertia between emitting and absorbing bodies [1].

In other article, Albert Einstein mentioned [1] (About the influence of gravity on the spread of light) (Über den Einfluss der Schwerkraft auf die Ausbreitung des Lichtes): "With respect to a gravitational system K with gravity $\mathrm{g}$, as well as with respect to an accelerated system $\mathrm{K}$ ' with acceleration $\mathrm{a}=-\mathrm{g}$ and without gravitational field, the material points, move according to the equations: $\frac{d^{2} x_{y}}{d t^{2}}=0 \quad \frac{d^{2} y_{y}}{d t^{2}}=0 \quad \frac{d^{2} z_{y}}{d t^{2}}=-g \quad\left(\mathrm{a}_{\mathrm{y}}=-\mathrm{g}\right)$ 
For the K' system, it follows directly from the Galileo principle, but for the $\mathrm{K}$ system, at rest in a homogeneous gravitational field, it follows from the experience that all bodies in a similar field are equally and uniformly accelerated with the same acceleration of the gravity. The very satisfactory conclusion is reached that the systems $\mathrm{K}$ and $\mathrm{K}^{\prime}$ are exactly equivalent from the physical point of view. The system $\mathrm{K}$ can be considered equally well to be in a space free of gravitational fields or in a uniformly accelerated system. This hypothesis of equivalence physics prohibits us from talking of the absolute acceleration of the reference frame, in the same way that the usual theory of relativity prohibits us from talking about the absolute speed of a system. Systems $\mathrm{K}$ and $\mathrm{K}^{\prime}$ are equivalent for all physical processes. That is, the laws of nature with respect to $\mathrm{K}$ are in complete agreement with the laws of nature with respect to $\mathrm{K}^{\prime \prime}$.

If a rocket rises with constant acceleration a (system $\mathrm{K}^{\prime}$ in a place where there is no gravitational field), then the objects do not appear to float, but rather rush in the opposite direction to the acceleration as in a planet with gravity [4]. For an observer in a place (system K) where there is a gravitational field with the same acceleration of the rocket, the results of the physical experiments carried out will be identical to those of the accelerated rocket. All the phenomena observed by the observer in the accelerated system are identical to those he will find in a gravitational field. Then, the effects of acceleration cannot be distinguished from those of gravitation.

Therefore, an accelerated system $\mathrm{K}^{\prime}$ is equivalent to a gravitational system $\mathrm{K}$. This constitutes the principle of equivalence: a fundamental principle in general relativity [4]. This equivalence principle applies to all reference frames. Besides, Albert Einstein asked the next question: "Is there a decrease in the gravitational mass corresponding to this decrease in inert mass?. If there is not, then a body would fall into the gravitational field with variable acceleration depending on the energy it contains [1]".

The inertial mass is equal to the gravitational mass because of the equivalence between the accelerated systems with the gravitational system [4]. If the gravitational mass were different from the inertial mass, different objects would have different accelerations in a gravitational field. But in a gravitational field, all objects have the same acceleration.

Then, it is concluded that the inertial mass is equal to the gravitational mass [1]. As the inertial mass is equal to the gravitational mass, the increase or decrease in the inertial mass corresponds to the increase or decrease of the gravitational mass due the absorption or emission of the gravitational energy. It constitutes the main postulate of General Relativity.

In resume, accelerated system and the gravitational system are equivalents, the principle of relativity is applicable to all systems and the inertial mass is equal to the gravitational mass. In the same article, Albert Einstein wrote: "I tried to answer the question of whether the propagation of light is influenced by gravitation. I now see that one of the most important consequences of my first treatment can be tested experimentally (curvature of space-time). Indeed, from the theory presented here, it follows that the light rays that pass near the Sun are deflected by the gravitational field of the Sun, so that the angular distance between the Sun and a fixed star that appears close to the Sun is apparently increases by almost an arc second (from 0,83 arc second to 1,75 arc second) [1]". 
If a ray of light enters to an accelerated rocket (where there is no gravitational field), the observer see that light has a curved path due the rocket advances rapidly while the light travels. For other observer out of the rocket, the light moves in a straight line [4]. Thus, the light is bent in an accelerated system. If the light is bent in an accelerated system and due the equivalence of the accelerated $\left(\mathrm{K}^{\prime}\right)$ and the gravitational $(\mathrm{K})$ systems, then the light is also bent in a gravitational system. It was one of the important results predicted by the Einstein Theory: Light is bent because Space-Time is curved in the presence and in the vicinity of a large mass with an intense gravitational field.

Thus, a star in a certain position will appear as if it were in another position because the light is deflected when passing near the Sun [5]. This confirmed the principle of equivalence: the equivalence between accelerated systems and gravitational fields. The General Theory of Relativity explains how space-time is curved due to the presence of a large mass or large concentration of matter. At this theory, the gravitational phenomena are not explained in terms of attractive forces, but they are related to the geometry of the Universe where the Euclidean geometry cannot be applied. The Geometry used to explain this curvature of the space time is the Riemann geometry [4] [5]. At this research, firstly it is presented and demonstrated the real gravitational interaction from the experiment of Cavendish. Then, it is showed a research for the bond total energy and energy emission for a particle orbiting a large object and for a binary star from a classic point of view. Then, it is demonstrated by theory, calculation and result the discovered formula which describe the mass of a particle which emits gravitational energy. The formula is in agreement at the classic limit for the bond energy for the particle orbiting a large object [6] and for a binary star [8]. Also, this formula is in agreement with the General Relativity Theory. Besides, the formula for the gravitational redshift, wavelength, light velocity, time measurement, and decrease distance in a gravitational field are demonstrated. In addition, the formula of the light velocity is tested for the light deflection passing close the Sun. Also, it is tested the formula of the decrease distance for the Perihelion Precession of Mercury. The results are in agreement with the experimental results with high accuracy.

\section{Experiment of Cavendish}

The objective of the Cavendish Experiment is to demonstrate the real gravitational interaction between objects with mass.

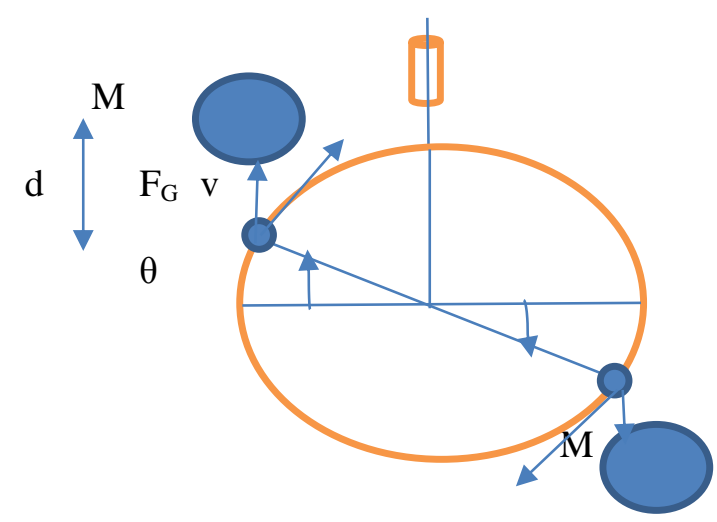

Fig.1 Cavendish Experiment: torsion balance with a light rod with two spheres mass $m$ which are attracted by the two spheres M due the Gravitational Force 
As the Newton Theory has established, the gravitational force is proportional to the product of the masses and inversely proportional to the square of the distance between them. During this time, it was unknown the gravitational constant $\mathrm{G}$ and the mass of the Earth. Cavendish used a torsion balance (torsion pendulum) at his famous experiment [9]. The experiment uses a torsion balance which consists of a light rod with two spheres of mass $\mathrm{m}$ at its ends which remain horizontal as it is showed at the figure.

Radius: $\mathrm{R} \quad \mathrm{M} \gg>\mathrm{m} \quad$ Circular Motion $\omega=\mathrm{d} \theta / \mathrm{dt} \quad \mathrm{v}=\mathrm{Rw} \quad \tau=-\mathrm{k} \theta$

Torque: $\tau \mathrm{k}$ : constant of the torque or Dynamic momentum of the thread

$\tau=\mathrm{F}_{\mathrm{t}} \mathrm{R} \quad \tau=\mathrm{ma}_{\mathrm{t}} \mathrm{R} \quad \tau=\mathrm{mR}^{2} \alpha \quad \mathrm{a}_{\mathrm{t}}=\mathrm{R} \alpha \quad F_{t}=F_{G}=G \frac{M m}{d^{2}}$

$\tau=2 \mathrm{mR}^{2} \alpha$ (for the two balls of mass $\mathrm{m}$ ) $\quad \tau=\mathrm{I} \alpha \quad \mathrm{I}=2 \mathrm{mR}^{2}$

If the system is moved a small angle $\theta$ from the equilibrium position and then left free, it will perform harmonic oscillations. The angular momentum $\mathrm{L}$ of the system is the sum of the angular moments of each ball.

$\mathrm{L}=\mathrm{pR} \quad \mathrm{L}=\mathrm{mvR} \quad \mathrm{L}=\mathrm{Iw}$

$\mathrm{L}=2 \mathrm{mvR} \quad \mathrm{L}=2 \mathrm{~m}(\mathrm{Rw}) \mathrm{w}=2 \mathrm{mR}^{2} \mathrm{w}=\mathrm{Iw} \quad \mathrm{I}=2 \mathrm{mR}^{2}$

$\frac{d L}{d t}=\frac{d I w}{d t}=I \frac{d w}{d t}=I \alpha=\tau$ (I: inertia: constant and independent of time).

$\frac{d L}{d t}=\tau \quad \tau=\mathrm{r} \times \mathrm{F} \quad \tau=\mathrm{I} \alpha \quad \alpha=\frac{d w}{d t} \quad \alpha=\frac{d^{2} \theta}{d t^{2}}$

$\frac{d L}{d t}=\tau \quad \mathrm{L}=2 \mathrm{mR}^{2} \mathrm{w} \quad \tau=-\mathrm{k} \theta \quad \alpha:$ the angular acceleration

$2 \mathrm{~m} R^{2} \frac{d w}{d t}=-\mathrm{k} \theta \quad 2 \mathrm{~m} R^{2} \alpha=-\mathrm{k} \theta \quad \alpha=\frac{-\mathrm{k} \theta}{2 \mathrm{~m} R^{2}} \quad \alpha=-\omega^{2} \theta$

$\omega=\sqrt{\frac{\mathrm{k}}{2 \mathrm{~m} R^{2}}} \quad T=\frac{2 \pi}{\omega} \quad \mathrm{T}=\sqrt{\frac{8 \pi^{2} \mathrm{~m} R^{2}}{\mathrm{k}}} \quad \mathrm{T}=2 \pi \sqrt{\frac{\mathrm{I}}{\mathrm{k}}}$

$2 \mathrm{~m} R^{2} \frac{d w}{d t}=-\mathrm{k} \theta \quad w=\frac{d \theta}{d t}$

$2 \mathrm{~m} R^{2} \frac{d^{2} \theta}{d t^{2}}=-\mathrm{k} \theta \quad \frac{d^{2} \theta}{d t^{2}}+\frac{k}{2 \mathrm{~m} R^{2}} \theta=0$

$\frac{d^{2} \theta}{d t^{2}}+\omega^{2} \theta=0$

Also, this differential equation is possible to obtain in this form:

$\tau=\mathrm{I} \alpha \quad \tau=-\mathrm{k} \theta$

$\mathrm{I} \alpha=-\mathrm{k} \theta \quad \alpha=\frac{d^{2} \theta}{d t^{2}}$

$\frac{d^{2} \theta}{d t^{2}}+\frac{k}{\mathrm{I}} \theta=0 \quad \mathrm{I}=2 \mathrm{mR}^{2}$

$\frac{d^{2} \theta}{d t^{2}}+\frac{k}{2 \mathrm{~m} R^{2}} \theta=0 \quad \omega=\sqrt{\frac{\mathrm{k}}{2 \mathrm{~m} R^{2}}}$ 
$\frac{d^{2} \theta}{d t^{2}}+\omega^{2} \theta=0$

$\theta=\theta_{o} \operatorname{sen}\left(\omega t+\frac{\pi}{2}\right) \quad$ (harmonic oscillations)

$w=\frac{d \theta}{d t}=\omega \theta_{o} \cos \left(\omega t+\frac{\pi}{2}\right) \quad$ angular velocity

$\alpha=\frac{d^{2} \theta}{d t^{2}}=-\omega^{2} \theta_{o} \operatorname{sen}\left(\omega t+\frac{\pi}{2}\right) \quad$ angular acceleration

The data of the Cavendish Experiment are:

$M=158 \mathrm{~kg}, \mathrm{~m}=0,73 \mathrm{~kg}, \mathrm{R}=0,90 \mathrm{~m}, \mathrm{~d}=0,23 \mathrm{~m}, \mathrm{~T}=420 \mathrm{~s}, \theta=0,000987 \mathrm{Rad}$

$\mathrm{k}=\frac{8 \pi^{2} \mathrm{~m} R^{2}}{T^{2}} \quad \mathrm{k}=2,65 \times 10^{-4} \mathrm{~N} \mathrm{~m}$

The gravitational forces due to the balls of mass M (located at a distance $\mathrm{d}$ of the balls of mass $\mathrm{m}$ ) are applied to each sphere $m$. The system will reach a new equilibrium state corresponding to a small angle $\theta_{\mathrm{o}}$ [9]. At this angle, the moment of these forces exert is balanced with the torsion. It shows the real gravitational force between the balls.

$\tau=\mathrm{F}_{\mathrm{t}} \mathrm{R} \quad F_{t}=F_{G}=G \frac{M m}{d^{2}}$

$2 \mathrm{~F}_{\mathrm{G}} \mathrm{R}=\mathrm{k} \theta$ (for the two balls of mass $\mathrm{m}$ )

$F_{G}=\frac{k \theta}{2 R} \quad F_{G}=G \frac{M m}{d^{2}}$

$G \frac{M m}{d^{2}}=\frac{k \theta}{2 R} \quad G=\frac{k \theta d^{2}}{2 R M m}$

$\mathrm{G}=6,64 \times 10^{-11} \mathrm{~N} \mathrm{~m}^{2} / \mathrm{kg}^{2}$

Besides, Cavendish obtained the value of the Earth Mass [9] as follows:

$G \frac{M_{T m}}{R_{T}{ }^{2}}=m g \quad G \frac{M_{T}}{R_{T}{ }^{2}}=g \quad$ (g: gravity at the Earth, this value changes depending of the deep inside of the Earth, $\mathrm{R}_{\mathrm{T}}$ : Radius of the Earth)

$M_{T}=\frac{g R_{T}{ }^{2}}{G} \quad \mathbf{M}_{\mathrm{T}}=\mathbf{5 , 9 8} \times \mathbf{1 0} \mathbf{2 4}^{24} \mathbf{k g}$

The density of the Earth is calculated as follows:

$\rho_{T}=\frac{M_{T}}{\frac{4}{3} \pi R_{T}{ }^{3}} \quad \rho_{T}=\frac{3 g}{4 \pi G R_{T}} \quad \rho_{\mathrm{T}}=5,51 \times 10^{3} \mathrm{~kg} / \mathrm{m}^{3}$

\section{A Particle Orbiting an Almost Static Massive Planet or Sun}

The mass of the particle is $\mathrm{m}$ and the mass of the massive planet or Sun is M. The particle moves around the planet in a circular motion with radius $\mathrm{R}$. If the planet moves around the Sun, the exact motion is an ellipse as Kepler has established. 


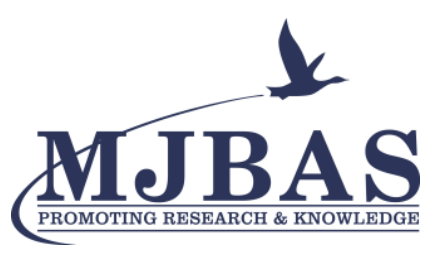

Mediterranean Journal of Basic and Applied Sciences (MJBAS)

Volume 4, Issue 3, Pages 42-69, July-September 2020

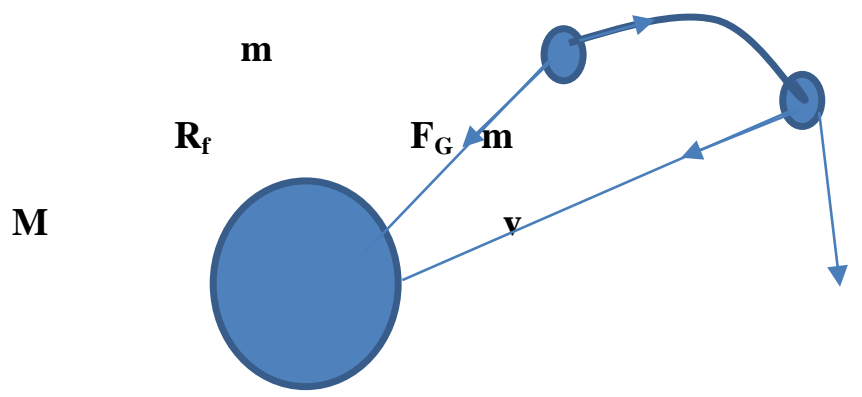

Fig.2 A particle orbiting an almost massive Planet or Sun due the Gravitational Force.

The gravitational force between the particle and the planet gives out the Gravitational Potential Energy. Besides, the particle emits gravitational energy due the presence of a massive planet near the particle. Then, the mass of the particle decreases due the emission of the gravitational energy. As result of it, the particle changes a little its trajectory by decreasing a little the distance $\mathrm{R}$ with the planet. Then, the particle starts to move in a circular or elliptical motion (rotational motion) around the massive planet due the initial velocity of the particle. It is the case of the planetary system moving or orbiting around its massive star. The effect of the little change of the trajectory is possible to measure at the motion of Mercury (Perihelion Precession).

\subsection{Variant Mass for a Particle due the Gravitational Potential Energy}

$\mathrm{dU}=-\mathrm{dW} \quad \mathrm{dW}=\mathrm{FdR} \quad \mathrm{F}=-\mathrm{G} \frac{\mathrm{Mm}}{\mathrm{R}^{2}}$

The gravitational potential energy is equal to the negative work done by the gravitational force because it is necessary to gives energy or to do work on the system to separate the particle $\mathrm{m}$.

$c^{2} d m=d U$

$c^{2} d m=\mathrm{G} \frac{\mathrm{Mm}}{\mathrm{R}^{2}} d R$

$\frac{\mathrm{dm}}{\mathrm{m}}=\frac{\mathrm{GM}}{\mathrm{c}^{2}} \frac{\mathrm{dR}}{\mathrm{R}^{2}}$

$\ln \frac{m}{m_{o}}=-\frac{G M}{c^{2}}\left(\frac{1}{R}-\frac{1}{R_{o}}\right)$

The reference point is arbitrary for the gravitational potential energy. It is chosen the point where the force is 0 at $\mathrm{R}_{\mathrm{o}}=\infty$.

$\boldsymbol{m}=\boldsymbol{m}_{o} e^{\frac{-G M}{c^{2}}\left(\frac{1}{R}\right)}($ Variant Mass due the Gravitational Potential Energy)

$e^{\frac{-G M}{c^{2}}\left(\frac{1}{R}\right)}=1-\frac{G M}{c^{2}}\left(\frac{1}{R}\right)+\frac{1}{2}\left[\frac{G M}{c^{2}}\left(\frac{1}{R}\right)\right]^{2}+\ldots$

$\frac{G M m}{R^{2}}=m \frac{v^{2}}{R} \quad \frac{G M}{R}=v^{2}$

$G M\left(\frac{1}{R}\right) \ll c^{2} \quad \mathrm{v}^{2}<<\mathrm{c}^{2} \quad \mathrm{v}<<\mathrm{c}$

$m=m_{o}\left[1-\frac{G M}{c^{2}}\left(\frac{1}{R}\right)\right] \quad$ (classic approach for the mass) 
$m c^{2}=m_{o} c^{2}-\frac{G M m_{0}}{R} \quad \mathrm{mc}^{2}=\mathrm{m}_{\mathrm{o}} \mathrm{c}^{2}+\mathrm{U} \quad \mathrm{mc}^{2}-\mathrm{m}_{\mathrm{o}} \mathrm{c}^{2}=\mathrm{U}$

$U=-\frac{G M m_{o}}{R}$ (classic approach for the gravitational potential energy)

The gravitational potential energy $\mathrm{U}$ is given by the next expression:

$\mathrm{U}=\mathrm{mc} \mathrm{c}^{2}-\mathrm{m}_{\mathrm{o}} \mathrm{c}^{2}$

$\mathrm{U}=m_{o} c^{2}\left(e^{\frac{-G M}{c^{2}}\left(\frac{1}{R}\right)}-1\right)$ (Gravitational Potential Energy)

$G M\left(\frac{1}{R}\right) \ll c^{2} \quad \mathrm{v}<<\mathrm{c}$

$\boldsymbol{U}=-\frac{\boldsymbol{G M} \boldsymbol{m}_{\boldsymbol{o}}}{\boldsymbol{R}}$ (which agrees with the classic approach)

$\mathrm{U}$ increases (less negative) when $\mathrm{R}$ increases. If we want to separate the particle $\mathrm{m}$ and the planet $\mathrm{M}$, it is necessary to apply an external force or to gives an additional energy to the system. The work done by this force produces an increase of the gravitational potential energy. Part of the work done by this force or the additional energy given produces a decrease of the kinetic energy and the particle has less velocity.

$\mathrm{U}$ decreases (more negative) when $\mathrm{R}$ decreases. If we want to reduce the distance of the particles $\mathrm{m}$ and the planet $M$, it is necessary that the particle $m$ emits energy (emission as gravitational energy). The emission of this energy produces a decrease of the gravitational potential energy. It produces also an increase of the kinetic energy and the particle has more velocity. If the particle changes a small distance near the surface of the Earth (R decreases) with radius $\mathrm{R}_{\mathrm{M}}$, we obtain the following for the gravitational potential energy: $\Delta U=-G M m_{o}\left(\frac{1}{R_{f}}-\frac{1}{R_{i}}\right)$

$\Delta U=G M m_{o}\left(\frac{R_{f}-R_{i}}{R_{f} R_{i}}\right) \quad \mathrm{R}_{\mathrm{f}}-\mathrm{R}_{\mathrm{i}} \approx-\Delta \mathrm{y} \quad \mathrm{R}_{\mathrm{f}} \mathrm{R}_{\mathrm{i}} \approx \mathrm{R}_{\mathrm{T}}^{2} \quad g=G \frac{M}{R_{T}^{2}}$

$\Delta \mathrm{U}=-\mathrm{m}_{\mathrm{o}} \mathrm{g} \Delta \mathrm{y}$ (the gravitational potential energy inside of the Earth).

\subsection{Variant Mass for a Particle due to the Kinetic Energy}

$c^{2} d m=d W$

$c^{2} d m=\tau d \theta$

$c^{2} d m=\frac{d L}{d t} d \theta \quad$ where $\mathrm{w}=\mathrm{d} \theta / \mathrm{dt}$

$c^{2} d m=w d L \quad \mathrm{~L}=\mathrm{Iw}$

$c^{2} d m=w d(I w)$

It depends of the time (no constant) and the inertial system (unless the body rotates around one of the main axes of inertia). It is possible to apply the equations of motion in a non-inertial system formed by the main axes of inertia of the solid. Then, I is constant at this case.

Nevertheless, It changes in our case due the mass increases (for relativistic velocities). 
$c^{2} d m=w^{2} d I+I w d w \quad \mathrm{I}=\mathrm{mR}^{2}$ (Inertia for a particle of mass $\mathrm{m}$ )

$c^{2} d m=w^{2} R^{2} d m+m R^{2} w d w$

$\left(c^{2}-w^{2} R^{2}\right) d m=m R^{2} w d w$

$\frac{d m}{m}=\frac{R^{2} w}{\left(c^{2}-w^{2} R^{2}\right)} d w$

$\int_{m_{o}}^{m} \frac{d m}{m}=\int_{0}^{w} \frac{R^{2} w}{\left(c^{2}-w^{2} R^{2}\right)} d w$

$u=c^{2}-w^{2} R^{2} \quad d u=-2 R^{2} w d v$

$\int_{m_{o}}^{m} \frac{d m}{m}=\int_{c^{2}}^{c^{2}+w^{2} R^{2}}-\frac{d u}{2 u}$

$\ln (\mathrm{m})-\ln \left(\mathrm{m}_{\mathrm{o}}\right)=(-1 / 2)\left[\ln \left(\mathrm{c}^{2}-\mathrm{w}^{2} \mathrm{R}^{2}\right)-\ln \left(\mathrm{c}^{2}\right)\right]$

$\ln \left(\mathrm{m} / \mathrm{m}_{\mathrm{o}}\right)=\ln \left[\left(\mathrm{c}^{2}-\mathrm{w}^{2} \mathrm{R}^{2}\right) / \mathrm{c}^{2}\right]^{-1 / 2}$

$\mathrm{m} / \mathrm{m}_{\mathrm{o}}=\left[\left(\mathrm{c}^{2}-\mathrm{w}^{2} \mathrm{R}^{2}\right) / \mathrm{c}^{2}\right]^{-1 / 2}$

$m=\frac{m_{o}}{\sqrt{\left(1-\frac{w^{2} R^{2}}{c^{2}}\right)}} \quad$ (mass of a particle with relativistic velocities) $\mathbf{v}=\mathbf{R w}$

$m=\frac{m_{o}}{\sqrt{1-\frac{v^{2}}{c^{2}}}} \quad \mathrm{v}:$ tangential velocity

At this formula, when the velocity $\mathrm{v}$ or $\mathrm{w}$ is increasing, the relativistic mass $\mathrm{m}$ is increasing. If $\mathrm{v}$ approximates to the light velocity $v \approx c$, then the value of mass is approaching infinity. Then, the velocity $v$ can never get the light velocity because it implies that the mass increases to the $\infty$.

$m=m_{o}\left(1+\frac{1}{2} \frac{R^{2} w^{2}}{c^{2}}+\frac{3}{8} \frac{R^{4} w^{4}}{c^{4}}+\cdots\right)$

$m=m_{o}\left(1+\frac{1}{2} \frac{R^{2} w^{2}}{c^{2}}\right) \quad \mathrm{Rw}<<\mathrm{c} \quad \mathrm{v}<<\mathrm{c}$

$m c^{2}=m_{o} c^{2}+\frac{1}{2} \frac{m_{o} R^{2} w^{2}}{c^{2}}$ classic approach for the mass

$\mathrm{E}=\mathrm{E}_{\mathrm{o}}+\mathrm{E}_{\mathrm{R}}$

$\mathrm{E}_{\mathrm{R}}=\frac{1}{2} \frac{m_{0} R^{2} w^{2}}{c^{2}} \quad \mathrm{I}=\mathrm{m}_{\mathrm{o}} \mathrm{R}^{2}$

$E_{R}=\frac{1}{2} I w^{2}$ classic approach for the rotational kinetic energy

The rotational kinetic formula is obtained as follows:

$E_{R}=m c^{2}-m_{0} c^{2}$

$E_{R}=m_{o} c^{2}\left(\frac{1}{\sqrt{\left(1-\frac{w^{2} R^{2}}{c^{2}}\right)}}-1\right)$ rotational kinetic energy 
$\mathrm{Rw}<<\mathrm{c} \quad \mathrm{v}<<\mathrm{c}$

$E_{R}=\frac{1}{2} m_{o} R^{2} w^{2}$ ( which agrees with the classic approach).

For higher velocities (when $\mathrm{v}$ or $\mathrm{wR}$ is approaching to c), it is necessary an infinity rotational kinetic energy to bring the particle to those velocities. There is limit of the rotational velocity for the particle which is the light velocity. If the particle decreases the radius orbit, the velocity and the kinetic energy increases. There is a maximum velocity $\mathrm{v}$ when the particle has radius $\mathrm{R}=\mathrm{R}_{\mathrm{o}}$ where $\mathrm{R}_{\mathrm{o}}$ is the radius of the planet. It corresponds also to the maximum approach to the massive planet or $\mathrm{R}$ minimum.

\subsection{Total Variant Mass for a Particle which emits Gravitational Energy: Classic Approach}

$m c^{2}=m_{o} c^{2}-\left(\frac{G M m_{o}}{R}\right)$ decrease mass: gravitational potential energy

$m_{o} c^{2}-m c^{2}=\left(\frac{G M m_{o}}{R}\right)$

$m c^{2}=m_{o} c^{2}\left(1+\frac{1}{2} \frac{R^{2} w^{2}}{c^{2}}\right) \quad$ (increase mass for kinetic energy)

$m \frac{v^{2}}{R}=\frac{G M m}{R^{2}} \quad \frac{1}{2} m v^{2}=\frac{G M m}{2 R}$

$\frac{1}{2} I w^{2}=\frac{1}{2} m R^{2} w^{2}=\frac{1}{2} m v^{2}=\frac{G M m}{2 R}=K=E_{R}$

$m c^{2}=m_{o} c^{2}+\frac{1}{2} \frac{G M m_{o}}{R}$

$m_{o} c^{2}-m c^{2}=-\frac{1}{2} \frac{G M m_{o}}{R}$

Therefore, the total lost mass is given by adding the contribution of the gravitational potential energy with the kinetic contribution to the mass.

$m_{o} c^{2}-m c^{2}=\frac{G M m_{o}}{R}-\frac{1}{2} \frac{G M m_{o}}{R}$

$\Delta \mathrm{mc}^{2}=m_{o} c^{2}-m c^{2}=\left(\frac{G M m_{o}}{2 R}\right)$ total decreases mass of the particle

\subsection{Total Variant Energy for a Particle which emits Gravitational Energy: Classic Approach}

The total variant energy is given by the same expression of the total mass decreasing of the particle in a gravitational field because of the Energy Mass Law $E=m c^{2}$. It is due that the total energy correspond to the bond energy of the system which after is emitted as gravitational Energy and corresponds to the lost mass of the particle.

Therefore, it is obtained:

$m c^{2}=m_{o} c^{2}-\left(\frac{G M m_{o}}{2 R}\right)$

$\mathrm{E}=m c^{2}-m_{o} c^{2}$

$E=\left(-\frac{G M m_{o}}{2 R}\right)$ total bond energy of the system $M$ and $\mathbf{m}$ 
Other form to obtain the formula is as follows:

If $\mathrm{M} \gg>\mathrm{m}$ kinetic energy of the planet with mass $\mathrm{M} \mathrm{K}_{\mathrm{M}}$ is negligible

$\mathrm{mv}=\mathrm{Mv}_{\mathrm{M}} \quad \mathrm{v}_{\mathrm{M}}=(\mathrm{m} / \mathrm{M}) \mathrm{v}$

$K_{M}=\frac{1}{2} M v_{M}^{2}=\frac{1}{2} \frac{m^{2}}{M} v^{2}=\frac{m}{M} K$ where $K=\frac{1}{2} m v^{2}$, then $\mathrm{K}_{\mathrm{M}}$ is negligible

$\mathrm{E}=\mathrm{K}+\mathrm{U}$

$E=\frac{1}{2} m v^{2}-\frac{G M m}{R} \quad$ or $E=\frac{1}{2} I w^{2}-\frac{G M m}{R}$

$\frac{G M m}{R^{2}}=\frac{m v^{2}}{R} \quad \mathrm{~F}_{\mathrm{G}}=\mathrm{F}_{\mathrm{C}}$

$\frac{G M m}{2 R}=\frac{1}{2} m v^{2}=\frac{1}{2} I w^{2}=K \quad \mathrm{I}=\mathrm{mR}^{2}$

$\mathrm{K}=\frac{G M m}{2 R} \quad E=\frac{G M m}{2 R}-\frac{G M m}{R}$

$E=-\frac{G M m}{2 R} \quad$ Total Bond Energy of the System

The total bond energy is negative and equal to the kinetic energy in absolute value for the case of circular orbits. Also, the kinetic energy is positive and equal to the half of the potential energy in absolute value [7]. This total bond energy corresponds to the value of the lost mass.

In the case of elliptical orbits, the total energy [7] is given by:

$E=-\frac{G M m}{2 a}$ where a is the semi-major axis.

\section{Formula Development of the Total Mass and Energy of the Particle in a Gravitational Field}

$$
\begin{aligned}
& c^{2} d m=\frac{d p}{d t} d s+\frac{G M m}{r^{2}} d r \\
& c^{2} d m=\frac{d(m v)}{d t} d s+\frac{G M m}{r^{2}} d r \\
& c^{2} d m=m v d v+v^{2} d m+\frac{G M m}{r^{2}} d r \\
& c^{2} d m-v^{2} d m=m v d v+\frac{G M m}{r^{2}} d r \\
& \frac{G M m}{r^{2}}=m \frac{v^{2}}{r} \\
& \frac{G M}{r}=v^{2} \\
& -\frac{G M}{2 r^{2}} d r=v d v \\
& c^{2} d m-\frac{G M}{r} d m=-\frac{G M m}{2 r^{2}} d r+\frac{G M m}{r^{2}} d r
\end{aligned}
$$


$\left(c^{2}-\frac{G M}{r}\right) d m=\frac{G M m}{2 r^{2}} d r$

$\frac{d m}{m}=\frac{\frac{G M}{2 r^{2}}}{\left(c^{2}-\frac{G M}{r}\right)} d r$

$\ln \left(\frac{m}{m_{o}}\right)=\frac{1}{2} \ln \left(\frac{c^{2}-\frac{G M}{r}}{c^{2}-\frac{G M}{r_{o}}}\right) \quad \mathrm{m}=\mathrm{m}_{\mathrm{o}} \quad \mathrm{r}_{\mathrm{o}}=\infty$

$m=m_{o} \sqrt{\left(1-\frac{G M}{r c^{2}}\right)} \quad \frac{G M}{r}=v^{2}$

$m=m_{o} \sqrt{\left(1-\frac{v^{2}}{c^{2}}\right)}$ mass for a particle in a gravitational field

$\Delta \mathrm{m}=\mathrm{m}_{\mathrm{o}}-\mathrm{m}$

$\Delta m=m_{o}\left(1-\sqrt{\left(1-\frac{G M}{r c^{2}}\right)}\right.$ total lost mass of the particle

$\mathrm{GM} / \mathrm{r}<<\mathrm{c}^{2}$

$m=m_{o}\left(1-\frac{G M}{2 r c^{2}}\right)$

$m c^{2}=m_{o} c^{2}-\frac{G M m_{o}}{2 r}$

$\Delta \mathrm{m}=m_{o} c^{2}-m c^{2}=\left(\frac{G M m_{o}}{2 R}\right)$ as the total mass decreasing of the particle in a gravitational field for the classic approach.

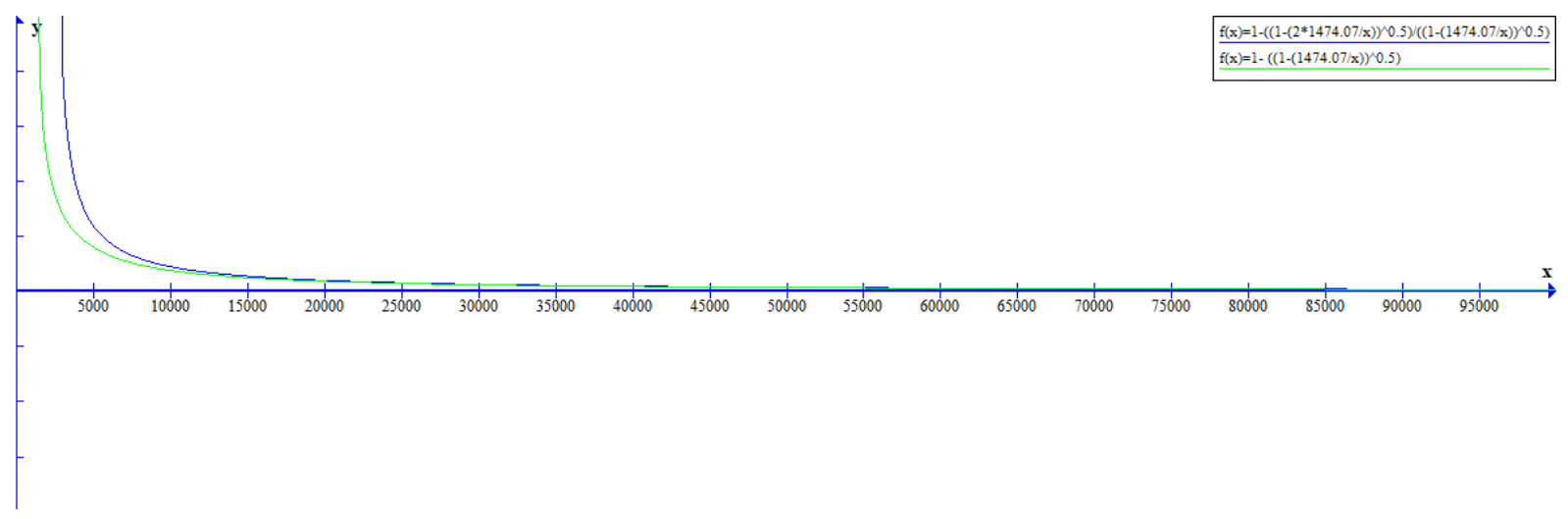

Fig.3 $\Delta \mathrm{m} / \mathrm{m}_{\mathrm{o}}$ versus the radius $\mathrm{r}$ from the mass development formula (green) and for the mass formula from General Relativity (blue)

The formula [3] for the mass given by the Relativity Theory is as follows:

$m=m_{o} \frac{\sqrt{1-2 \frac{G M}{r c^{2}}}}{\sqrt{1-\frac{G M}{r c^{2}}}}$

$\mathrm{GM} / \mathrm{r}<<\mathrm{c}^{2}$ 
$\sqrt{1-2 \frac{G M}{r c^{2}}} \approx 1-\frac{G M}{r c^{2}}$

$\left(1-\frac{G M}{r c^{2}}\right)^{-1 / 2} \approx 1+\frac{G M}{2 r c^{2}}$

$m=m_{o}\left(1-\frac{G M}{2 r c^{2}}\right) \quad$ (as the classic approach).

$m c^{2}=m_{o} c^{2}+E \quad \mathrm{E}=m c^{2}-m_{o} c^{2}$

$\mathrm{E}=-\frac{G M m_{o}}{2 r} \quad($ as the classic approach $)$.

$\Delta \boldsymbol{m} / \boldsymbol{m}_{\boldsymbol{o}}=\left(\mathbf{1}-\sqrt{\left(1-\frac{G M}{r c^{2}}\right)} \quad\right.$ Development mass formula

$\Delta \boldsymbol{m} / \boldsymbol{m}_{\boldsymbol{o}}=\left(\mathbf{1}-\frac{\sqrt{1-2 \frac{G M}{r c^{2}}}}{\sqrt{1-\frac{G M}{r c^{2}}}}\right) \quad$ General Relativity formula

Both formulas for the total mass decreasing of the particle agree at distances very far from the massive object and also at distances very near to the massive object with high accuracy. Then, both models: the Newton Approach with a gravitational field interaction and the Relativity Theory with a curvature of the space time are in agreement at the results for the total mass decreasing of the particle in a gravitational field.

For the total energy of the particle, it is obtained:

$\mathrm{E}=m c^{2}-m_{o} c^{2}$

$E=m_{o} c^{2}\left(\sqrt{\left(1-\frac{G M}{r c^{2}}\right.}-1\right)$ energy for a particle in a gravitational field

$\mathrm{GM} / \mathrm{r}<<\mathrm{c}^{2}$

$\mathrm{E}=-\frac{G M m_{o}}{2 r} \quad$ (as the classic approach)

The total mass energy lost is given by the formula demonstrated and it is equal in absolute value to the total bond energy of the system which is emitted as gravitational energy when the particle goes from one orbit to another orbit with less radius. The emission of the total gravitational energy E produces a lost mass of the particle $\mathrm{m}=\mathrm{E} / \mathrm{c}^{2}$.

If $\mathrm{R}_{\mathrm{f}}<\mathrm{R}_{\mathrm{i}} \Delta \mathrm{E}=E_{f}-E_{i}=\left(-\frac{G M m}{2 R_{f}}\right)-\left(-\frac{G M m}{2 R_{i}}\right)$ is negative, it is given out energy which means that the particle losses mass, the particle increases the kinetic energy and the velocity but decreases the gravitational potential energy (more negative). Part of the losses energy (gravitational energy emission) is given out by decreasing the potential energy and part by increasing the kinetic energy [7].

If $\mathrm{R}_{\mathrm{f}}>\mathrm{R}_{\mathrm{i}} \Delta \mathrm{E}$ is positive, which means that additional energy is given to the particle or additional work is done on the system, the particle decreases the kinetic energy and the velocity but increases the potential energy (less 
negative). Part of the work or additional energy is used to increase the potential energy and part to diminish the kinetic energy [7].

\section{Binary Stars}

If the particle and the planet have comparable and similar masses, then they start to move around each other and orbiting around their common barycenter (rotational motion) [8].

\subsection{Case $m_{1} \neq m_{2}$}

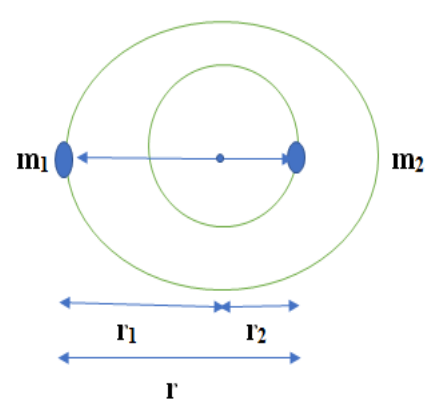

Fig.4 Binary Stars orbiting around their common barycenter due the Gravitational Force

It occurs in binary stars for example. Also, they emit gravitational energy and as a consequence, there is a decrease in their masses and the distance between them due this energy emission.

By considering the center of mass in $r=0$, it is obtained:

$\mathrm{m}_{1} \mathrm{r}_{1}=\mathrm{m}_{2} \mathrm{r}_{2} \quad \mathrm{r}_{1}=\mathrm{m}_{2} \mathrm{r}_{2} / \mathrm{m}_{1}$

$\mathrm{r}=\mathrm{r}_{1}+\mathrm{r}_{2}$

$\mathrm{r}=\left(\mathrm{m}_{2} \mathrm{r}_{2} / \mathrm{m}_{1}\right)+\mathrm{r}_{2} \quad \mathrm{r}=\left(\mathrm{m}_{1}+\mathrm{m}_{2}\right) \mathrm{r}_{2} / \mathrm{m}_{1}$

$\mathbf{r}_{2}=\left(\mathbf{m}_{1}\right) \mathbf{r} /\left(\mathbf{m}_{1}+\mathbf{m}_{2}\right)$

$\mathrm{r}_{2}=\mathrm{m}_{1} \mathrm{r}_{1} / \mathrm{m}_{2}$

$\mathrm{r}=\mathrm{r}_{1}+\mathrm{r}_{2} \quad \mathrm{r}=\mathrm{r}_{1}+\mathrm{m}_{1} \mathrm{r}_{1} / \mathrm{m}_{2}$

$\mathrm{r}=\left(\mathrm{m}_{1}+\mathrm{m}_{2}\right) \mathrm{r}_{1} / \mathrm{m}_{2}$

$\mathbf{r}_{1}=\left(\mathbf{m}_{2}\right) \mathbf{r} /\left(\mathbf{m}_{1}+\mathbf{m}_{2}\right)$

$K_{1}=\frac{1}{2} m_{1} v_{1}^{2} \quad \mathrm{v}_{1}=\mathrm{w}_{1} \mathrm{r}_{1} \quad \mathrm{w}_{1}=\mathrm{v}_{1} / \mathrm{r}_{1}$

$G \frac{m_{1} m_{2}}{r^{2}}=m_{1} \frac{v_{1}^{2}}{r_{1}}$

$G \frac{m_{2} r_{1}}{r^{2}}=v_{1}^{2} \quad G \frac{m_{2}^{2}}{r\left(m_{1}+m_{2}\right)}=v_{1}^{2}$

$w_{1}{ }^{2}=\frac{v_{1}^{2}}{r_{1}{ }^{2}}=G \frac{m_{2}}{r^{2} r_{1}} \quad w_{1}{ }^{2}=G \frac{\left(m_{1}+m_{2}\right)}{r^{3}}$ 
$K_{1}=\frac{1}{2} m_{1} v_{1}^{2}=\frac{1}{2} m_{1} G \frac{m_{2} r_{1}}{r^{2}}=\frac{1}{2} G \frac{m_{1} m_{2} r_{1}}{r^{2}}$

$\mathrm{r}_{1}=\left(\mathrm{m}_{2}\right) \mathrm{r} /\left(\mathrm{m}_{1}+\mathrm{m}_{2}\right)$

$K_{1}=\frac{1}{2} G \frac{m_{1} m_{2}}{r} \frac{m_{2}}{\left(m_{1}+m_{2}\right)}$ kinetic energy for $m_{1}$

$K_{2}=\frac{1}{2} m_{2} v_{2}^{2} \quad \mathrm{v}_{2}=\mathrm{w}_{2} \mathrm{r}_{2} \quad \mathrm{w}_{2}=\mathrm{v}_{2} / \mathrm{r}_{2}$

$G \frac{m_{1} m_{2}}{r^{2}}=m_{2} \frac{v_{2}^{2}}{r_{2}}$

$G \frac{m_{1} r_{2}}{r^{2}}=v_{2}^{2} \quad G \frac{m_{1}^{2}}{r\left(m_{1}+m_{2}\right)}=v_{2}^{2}$

$w_{2}{ }^{2}=\frac{v_{2}{ }^{2}}{r_{2}{ }^{2}}=G \frac{m_{1}}{r^{2} r_{2}} \quad w_{2}^{2}=G \frac{\left(m_{1}+m_{2}\right)}{r^{3}}$

$K_{2}=\frac{1}{2} m_{2} v_{2}{ }^{2}=\frac{1}{2} m_{2} G \frac{m_{1} r_{2}}{r^{2}}=\frac{1}{2} G \frac{m_{1} m_{2} r_{2}}{r^{2}}$

$\mathrm{r}_{2}=\left(\mathrm{m}_{1}\right) \mathrm{r} /\left(\mathrm{m}_{1}+\mathrm{m}_{2}\right)$

$K_{2}=\frac{1}{2} G \frac{m_{1} m_{2}}{r} \frac{m_{1}}{\left(m_{1}+m_{2}\right)}$ kinetic energy for $m_{2}$

$m_{1}$ and $m_{2}$ have different kinetic energies

$K_{T}=K_{1}+K_{2}=\frac{1}{2} G \frac{m_{1} m_{2}}{r} \frac{m_{2}}{\left(m_{1}+m_{2}\right)}+\frac{1}{2} G \frac{m_{1} m_{2}}{r} \frac{m_{1}}{\left(m_{1}+m_{2}\right)}$

$K_{T}=\frac{1}{2} G \frac{m_{1} m_{2}}{r}$

$U=-G \frac{m_{1} m_{2}}{r}$

Therefore, the total mechanical energy of the system of the binary stars which is given as emission of gravitational energy is:

$E=K_{T}+U=\frac{1}{2} G \frac{m_{1} m_{2}}{r}-G \frac{m_{1} m_{2}}{r}$

$E=-G \frac{m_{1} m_{2}}{2 r}$

It is the same total energy formula of a particle orbiting an almost static massive object. Each mass $\mathrm{m}$ has equal angular velocity, equal angular frequency and different kinetic energy with different linear velocities.

The mass of each star is as follows:

$m_{1}=m_{o 1} \sqrt{\left(1-\frac{v_{1}^{2}}{c^{2}}\right)} \quad G \frac{m_{o 2}{ }^{2}}{r\left(m_{o 1}+m_{o 2}\right)}=v_{1}{ }^{2}$

$m_{2}=m_{o 2} \sqrt{\left(1-\frac{v_{2}^{2}}{c^{2}}\right)} \quad G \frac{m_{o 1}{ }^{2}}{r\left(m_{o 1}+m_{o 2}\right)}=v_{2}{ }^{2}$ 
Then, each star has different lost mass given by $\Delta m=m_{01} c^{2}-m_{1} c^{2}$. Also, each star has different gravitational energy emission corresponding to the lost mass of the respective star. It is possible to obtain the total energy at the classic approach:

$$
\begin{aligned}
& m_{1}=m_{o 1}\left(1-\frac{v_{1}^{2}}{2 c^{2}}\right) \quad \mathrm{E}_{1}=\mathrm{m}_{1} \mathrm{c}^{2}-\mathrm{m}_{\mathrm{o} 1} \mathrm{c}^{2}=-\frac{v_{1}^{2}}{2} m_{o 1} \\
& m_{2}=m_{o 2}\left(1-\frac{v_{2}{ }^{2}}{2 c^{2}}\right) \quad \mathrm{E}_{2}=\mathrm{m}_{2} \mathrm{c}^{2}-\mathrm{m}_{02} \mathrm{c}^{2}=-\frac{v_{2}{ }^{2}}{2} m_{o 2} \\
& \mathrm{E}=\mathrm{E}_{1}+\mathrm{E}_{2}=-G \frac{m_{02}{ }^{2}}{r\left(m_{o 1}+m_{o 2}\right)} \frac{m_{o 1}}{2}-G \frac{m_{o 1}{ }^{2}}{r\left(m_{o 1}+m_{o 2}\right)} \frac{m_{o 2}}{2}
\end{aligned}
$$

$E=-G \frac{\boldsymbol{m}_{01} \boldsymbol{m o}_{2}}{2 r}$ as the classic result

\subsection{Case $m_{1}=m_{2}=m$}

$\mathrm{mr}_{1}=\mathrm{mr}_{2} \quad \mathrm{r}_{1}=\mathrm{r}_{2}$

Each mass $m$ orbits the same circle of radius $r_{1}=r_{2}$

$\mathrm{r}=\mathrm{r}_{1}+\mathrm{r}_{2}$

$r=2 r_{1} \quad r=2 r_{2} \quad r_{1}=r / 2 \quad r_{2}=r / 2$

For $\mathrm{m}_{1}$, it is obtained:

$G \frac{m m}{r^{2}}=m \frac{v_{1}^{2}}{r_{1}} \quad G \frac{m r_{1}}{r^{2}}=v_{1}{ }^{2} \quad G \frac{m}{2 r}=v_{1}^{2} \quad \mathrm{r}_{1}=\mathrm{r} / 2$

$w_{1}^{2}=\frac{v_{1}^{2}}{r_{1}^{2}}=G \frac{m}{r^{2} r_{1}}=G \frac{2 m}{r^{3}}$

$K_{1}=\frac{1}{2} m v_{1}^{2}$

$K_{1}=\frac{1}{2} m v_{1}^{2}=\frac{1}{2} m G \frac{m}{2 r}=G \frac{m^{2}}{4 r}$

For $\mathrm{m}_{2}$, it is obtained:

$G \frac{m m}{r^{2}}=m \frac{v_{2}{ }^{2}}{r_{2}} \quad G \frac{m r_{2}}{r^{2}}=v_{2}{ }^{2} \quad G \frac{m}{2 r}=v_{2}{ }^{2} \quad \mathrm{r}_{2}=\mathrm{r} / 2$

$w_{2}^{2}=\frac{v_{2}^{2}}{r_{2}{ }^{2}}=G \frac{m}{r^{2} r_{2}}=G \frac{2 m}{r^{3}}$

$K_{2}=\frac{1}{2} m v_{2}^{2}$

$K_{2}=\frac{1}{2} m v_{2}^{2}=\frac{1}{2} m G \frac{m}{2 r}=G \frac{m^{2}}{4 r}$

It is possible to obtain the same result, if we replace $m_{1}=m_{2}$ in the formula:

$K_{1}=\frac{1}{2} G \frac{m_{1} m_{2}}{r} \frac{m_{2}}{\left(m_{1}+m_{2}\right)} \quad K_{1}=G \frac{m^{2}}{4 r} \quad \mathrm{~m}_{1}=\mathrm{m}_{2}=\mathrm{m}$ 
$K_{2}=\frac{1}{2} G \frac{m_{1} m_{2}}{r} \frac{m_{1}}{\left(m_{1}+m_{2}\right)} \quad K_{2}=G \frac{m^{2}}{4 r}$

$K_{T}=K_{1}+K_{2}=2 G \frac{m^{2}}{4 r}$

$\boldsymbol{K}_{\boldsymbol{T}}=G \frac{m^{2}}{2 r} \quad \boldsymbol{U}=-G \frac{m^{2}}{r}$

Each mass $m$ has equal angular velocity, equal angular frequency, equal energy emission, equal linear velocity and equal kinetic energy. Thus, the total mechanical energy of the system of the binary stars is:

$E=K_{T}+U=G \frac{m^{2}}{2 r}-G \frac{m^{2}}{r}$

$E=-G \frac{m^{2}}{2 r}$

It is possible to obtain the total energy of the system from the formula:

$K_{T}=K_{1}+K_{2}=\frac{1}{2} G \frac{m_{1} m_{2}}{r} \frac{m_{2}}{\left(m_{1}+m_{2}\right)}+\frac{1}{2} G \frac{m_{1} m_{2}}{r} \frac{m_{1}}{\left(m_{1}+m_{2}\right)}$

$\boldsymbol{U}=-\boldsymbol{G} \frac{\boldsymbol{m}_{1} \boldsymbol{m}_{2}}{\boldsymbol{r}} \quad$ For $\mathrm{m}_{1}=\mathrm{m}_{2}=\mathrm{m}$ and $\mathrm{r}_{1}=\mathrm{r}_{2}=\mathrm{r} / 2 \quad \mathrm{r}=\mathrm{r}_{1}+\mathrm{r}_{2}$

$\boldsymbol{E}=\boldsymbol{K}_{\boldsymbol{T}}+\boldsymbol{U}=\mathbf{2} G \frac{m^{2}}{4 r}-\boldsymbol{G} \frac{m^{2}}{\boldsymbol{r}}$

$E=-G \frac{m^{2}}{2 r}$

The mass of each star is as follows:

$m_{1}=m_{o 1} \sqrt{\left(1-\frac{v_{1}^{2}}{c^{2}}\right)} \quad G \frac{m_{o}}{2 r}=v_{1}^{2} \quad \mathrm{~m}_{01}=\mathrm{m}_{02}=\mathrm{m}_{\mathrm{o}}$

$m_{2}=m_{o 2} \sqrt{\left(1-\frac{v_{2}^{2}}{c^{2}}\right)} \quad G \frac{m_{o}}{2 r}=v_{2}^{2}$

Then, each star has equal lost mass given by $\Delta \mathrm{m}=\mathrm{m}_{\mathrm{o}} \mathrm{c}^{2}-\mathrm{mc}^{2}$. Also, each star has equal gravitational energy emission. It is possible to obtain for the total energy at the classic approach:

$m_{1}=m_{o 1}\left(1-\frac{v_{1}^{2}}{2 c^{2}}\right) \quad \mathrm{E}_{1}=\mathrm{m}_{1} \mathrm{c}^{2}-\mathrm{m}_{\mathrm{o} 1} \mathrm{c}^{2}=-\frac{v_{1}^{2}}{2} m_{o 1}$

$m_{2}=m_{o 2}\left(1-\frac{v_{2}^{2}}{2 c^{2}}\right) \quad \mathrm{E}_{2}=\mathrm{m}_{2} \mathrm{c}^{2}-\mathrm{m}_{\mathrm{o} 2} \mathrm{c}^{2}=-\frac{v_{2}^{2}}{2} m_{o 2}$

$\mathrm{E}=\mathrm{E}_{1}+\mathrm{E}_{2}=-\boldsymbol{G} \frac{\boldsymbol{m}_{\boldsymbol{o}}}{\boldsymbol{r} \boldsymbol{r}} \frac{m_{o}}{2}-\boldsymbol{G} \frac{\boldsymbol{m}_{\boldsymbol{o}}}{\boldsymbol{2} \boldsymbol{r}} \frac{m_{o}}{2} \quad \mathrm{~m}_{\mathrm{o} 1}=\mathrm{m}_{\mathrm{o} 2}=\mathrm{m}_{\mathrm{o}}$

$E=-\boldsymbol{G} \frac{\boldsymbol{m}_{\boldsymbol{o}}{ }^{2}}{2 \boldsymbol{r}}$ as the classic result 


\section{Thought Experiment (Gedanken experiment)}

\subsection{Thought Experiment 1}

Gravitational Red Shift and Effects of the Gravitation on the time measure, frequency, wavelength and on the light velocity.

A uniform gravitational field $\mathrm{g}$ is directed downward in $\mathrm{K}$, with the photon emitted by an atom A falling a distance $\mathrm{d}$ through this field before it is absorbed by the detector $\mathrm{D}$ [5]. The system $\mathrm{K}$ ' is accelerated uniformly upwards with respect to $\mathrm{K}$ with $\mathrm{a}=\mathrm{g}$.
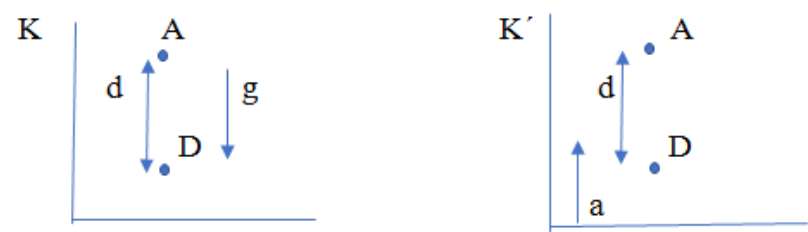

Fig.5 The system $\mathrm{K}$ with the gravitational field $\mathrm{g}$ and with the photon falling a distance $\mathrm{d}$ before it is absorbed by the detector $\mathrm{D}$. The same for the equivalent system $\mathrm{K}^{\prime}$ but with the acceleration $\mathrm{a}=\mathrm{g}$.

When the photon is emitted in the system $\mathrm{K}^{\prime}$, it has some velocity $\mathrm{u}$ in this system. The velocity of the detector when the photon reaches is $\mathrm{u}+\mathrm{at}$, where $\mathrm{t}$ is the photon's flight time: $\mathrm{t}$ is approximately $\mathrm{d} / \mathrm{c}$ and $\mathrm{a}=\mathrm{g}$.

The velocity of the detector absorbing it is $\mathrm{u}+\mathrm{gd} / \mathrm{c}$. If it is introduced the un-accelerated reference system $\mathrm{K}_{\mathrm{o}}$, with respect to which at the instant of light emission, $\mathrm{K}$ ' has not velocity, then $\mathrm{D}$, at the instant of arrival of the radiation at $\mathrm{D}$ has the velocity $v=\frac{g d}{c}$ with respect to $\mathrm{K}_{\mathrm{o}}[1]$. Indeed, the detector has an approximation velocity with respect to the emitter of $v=g d / c$, independent of $u$.

Therefore the received frequency $\mathrm{f}^{\prime}$ at the detector is greater than the emitted one $\mathrm{f}$. It is giving by the formula of the Doppler Effect:

$\frac{f^{\prime}}{f}=\sqrt{\frac{c+v}{c-v}}=\sqrt{\frac{c+\frac{g d}{c}}{c-\frac{g d}{c}}} \quad \frac{f^{\prime}}{f}=\sqrt{\frac{1+\frac{g d}{c^{2}}}{1-\frac{g d}{c^{2}}}}$

$\left(1+\frac{g d}{c^{2}}\right)^{1 / 2} \cong 1+\frac{g d}{2 c^{2}} \quad \mathrm{gd}<<c^{2}$

$\left(1-\frac{g d}{c^{2}}\right)^{-1 / 2} \cong 1+\frac{g d}{2 c^{2}} \quad \mathrm{gd}<<\mathrm{c}^{2}$

$\left(1+\frac{g d}{c^{2}}\right)^{1 / 2}\left(1-\frac{g d}{c^{2}}\right)^{-1 / 2}=\left(1+\frac{g d}{2 c^{2}}\right)\left(1+\frac{g d}{2 c^{2}}\right) \quad\left(\frac{g d}{2 c^{2}}\right)^{2} \approx 0 \quad$ for $\mathrm{gd}<<\mathrm{c}^{2}$

$\frac{f^{\prime}}{f} \cong 1+\frac{g d}{c^{2}} \quad f^{\prime} \cong f\left(1+\frac{g d}{c^{2}}\right)$ for system $\mathrm{K}^{\prime}$

$f^{\prime} \cong f\left(1+\frac{v}{c}\right)$ where $v=\frac{g d}{c}$ 
It is possible to obtain the same formula with the equivalence principle for the system $\mathrm{K}$ as follows: Let's consider that $\mathrm{A}$ and $\mathrm{D}$ are at rest and there is no Doppler effect which explains the increase in frequency. But, there is a gravitational field and this field acts on the photon [5].

It is possible to obtain the same formula with the equivalence principle for the system $\mathrm{K}$ where there is a gravitational field (A and D are at rest). It is given to the photon a gravitational mass equal to its inertial mass $\mathrm{E} / \mathrm{c}^{2}$. When falling a distance $\mathrm{d}$, the photon gains an energy $\left(\mathrm{E} / \mathrm{c}^{2}\right) \mathrm{gd}$. In quantum theory, the relationship for $\mathrm{E}$ is $\mathrm{E}=\mathrm{hf}$. It can be shown that $\mathrm{E}$ is proportional to $\mathrm{f}$ and that from the relativistic transformation of energy and momentum. Thus, it is obtained: $\Delta \mathrm{m}=\mathrm{E} / \mathrm{c}^{2}=\mathrm{hf} / \mathrm{c}^{2}$.

The energy of the photon being absorbed at $\mathrm{D}$ is its initial emission energy plus the energy gained by falling from A. This absorption energy in $\mathrm{D}$ is :

$\mathrm{E}^{\prime}=\mathrm{E}+\left(\mathrm{E} / \mathrm{c}^{2}\right) \mathrm{gd} \quad E^{\prime}=E\left(1+\frac{g d}{c^{2}}\right) \quad \mathrm{E}^{\prime}=\mathrm{hf}^{\prime}$ $h f^{\prime}=h f+\frac{h f g d}{c^{2}} \quad \frac{f^{\prime}}{f} \cong 1+\frac{g d}{c^{2}}$ $f^{\prime} \cong f\left(1+\frac{g d}{c^{2}}\right)$ for system $\mathrm{K}$ and equal to the system $\mathrm{K}^{\prime}$.

Therefore, it is necessary to add to the energy E the potential energy due the gravity. It probes also that the increase/decrease of the inertial mass in the accelerated system $\mathrm{K}^{\prime}$ corresponds to the increase/decrease of the gravitational mass in the gravitational system $\mathrm{K}$.

Thus, when light falls in a gravitational field at the same direction of the gravity acceleration, the light acquires greater energy and frequency, its wavelength decreases and it is said to deviate towards the blue. If the emitter and detector had been reversed and light rises in opposite direction to the gravity acceleration, it is concluded that the energy and frequency of the light decrease, its wavelength increases and it is said to drift towards the red. Thus, if the photon instead of descending from $\mathrm{A}$ to $\mathrm{D}$ in the accelerated system, it ascends from $\mathrm{D}$ to $\mathrm{A}$, the formula is:

$f^{\prime} \cong f\left(1-\frac{g d}{c^{2}}\right)$ light rises in opposite direction to the gravitational field

The value of the gravitational potential is replaced and it is obtained: $\quad E^{\prime}=E\left(1+\frac{\Phi}{c^{2}}\right)$

$$
\begin{array}{ll}
\mathrm{U}=\mathrm{m}_{\mathrm{o}} \mathrm{gd} & U=-\frac{G M m_{0}}{R} \\
\frac{G M m_{0}}{R}=\operatorname{mogd} & \frac{G M}{R}=\mathrm{gd}=\Phi
\end{array}
$$

$f^{\prime} \cong f\left(1-\frac{G M}{R c^{2}}\right)$ gravitational redshift for the light

Where $\mathrm{M}$ is the mass of the planet or Sun and $\mathrm{R}$ is the respective radius. The minus sign is because the photon losses energy to pass near the gravitational field of the Sun or the Planet. This is known as gravitational red shift, because light in the visible part of the spectrum will be shifted toward the red end [5]. 
By using the variant Mass due the Gravitational Potential Energy (3.1), it is obtained: $\quad \boldsymbol{m}=\boldsymbol{m}_{\boldsymbol{o}} \boldsymbol{e}^{\frac{-\boldsymbol{G M}}{c^{2}}\left(\frac{1}{R}\right)}$

$G M\left(\frac{1}{R}\right) \ll c^{2} \quad \mathrm{v}^{2}<<\mathrm{c}^{2} \quad \mathrm{v}<<\mathrm{c}$

$m=m_{o}\left[1-\frac{G M}{c^{2}}\left(\frac{1}{R}\right)\right]$

$m c^{2}=m_{o} c^{2}\left[1-\frac{G M}{c^{2}}\left(\frac{1}{R}\right)\right]$

For the light, it is necessary to replace $\mathrm{mc}^{2}=\mathrm{hf}$ :

$f^{\prime} \cong f\left(1-\frac{G M}{R c^{2}}\right)$ gravitational redshift for the light

It is possible to have the next question due the gravitational redshift: if there is constant transmission of light from A to $\mathrm{D}$, how can any other number of periods per second other than that emitted at A reach D?. The answer is because we cannot consider $\mathrm{f}$ or $\mathrm{f}$ ' simply as frequencies (as the number of periods per second) since we have not yet determined the time in the system $\mathrm{K}$. What $\mathrm{f}$ denotes is the number of periods per second with reference to the unit of time of the clock $\mathrm{U}$ in $\mathrm{A}$, while $\mathrm{f}^{\prime}$ denotes the number of periods per second with reference to the identical clock in D [1].

Nothing forces us to suppose that U-clocks at different gravitational potentials must be considered to be running at the same rate. On the contrary, we must certainly define the time in the gravitational system $\mathrm{K}$ in such a way that the number of wave crests and valleys between $\mathrm{A}$ and $\mathrm{D}$ is independent of the absolute value of time. Thus, the two clocks at $\mathrm{A}$ and $\mathrm{D}$ measures the time at the same rate. It means that If we measure the time in $\mathrm{D}$ with the clock $\mathrm{U}$, then we must measure the time in A with a clock running $\left(1-\frac{G M}{R c^{2}}\right)$ times slower than the clock $\mathrm{U}: t_{A}=t_{D}(1-$ $\frac{G M}{R c^{2}}$ ) when compared to $\mathrm{U}$ in $\mathrm{D}$ and in the same place [1]. Then, the relation of the both times is as follows:

$t_{D}=t_{A} /\left(1-\frac{G M}{R c^{2}}\right) \quad t^{\prime}=t /\left(1-\frac{G M}{R c^{2}}\right)$

Then, when we measure by this clock, the frequency of the light ray in its emission at A is equal to: $f\left(1-\frac{G M}{R c^{2}}\right)$ (less count of number of wave crests and valleys due the time dilation measures in A), and therefore it is equal to the frequency $f$ ' of the same light ray upon arrival at the detector $D$.

Thus, it is necessary to use $\mathrm{U}$ clocks of different constitution to measure time in places of different gravitational potential. If we want to measure time in a place that, with respect to the origin of coordinates, has the gravitational potential $\Phi$, it is necessary to use a clock that (when brought to the origin of coordinates) runs $\left(1-\frac{G M}{R c^{2}}\right)$ times slower than the clock used to measure time at the origin of coordinates.

Also, the formula for the decreased distance $\mathrm{d}$ of a particle orbiting a Planet due the gravitational energy emission is as follows:

$d^{\prime}=d\left(1-\frac{G M}{r c^{2}}\right)$ 
The wavelength is given by the next formula:

$\lambda^{\prime}=\lambda\left(1-\frac{G M}{R c^{2}}\right) \quad \lambda=\lambda_{\mathrm{G}}$ : increased wavelength due the gravitational potential.

The light velocity $\mathrm{v}^{\prime}=\lambda^{\prime} \mathrm{f}^{\prime}$ is obtained by replacing the respective formula $\lambda^{\prime}$ and $\mathrm{f}^{\prime}$ or $v^{\prime}=\frac{d^{\prime}}{t^{\prime}}$ :

$v^{\prime}=v_{o}\left(1-\frac{G M}{R c^{2}}\right)^{2}$

$v^{\prime} \cong v_{o}\left(1-2 \frac{G M}{R c^{2}}\right)$ light velocity in a gravitational field $\Phi$

It is also known as the refraction index for the light [3].

The variation velocity is because the different gravitational potential between different places. It is consequence of the time dilation of the place with gravitational field. The light has lower velocity in a place with gravitational velocity than in a place without the gravitational field [1].

This seems to contradict the principle of constancy of the speed of light. But there is no contradiction, because this light velocity variation is due the gravitational field. The light velocity remains constant at the place where there is the same gravitational potential but with different value in relation with other location where there is other gravitational potential. In resume, in a gravitational field, the light has lower velocity, lower rates of the clock-time (less time measured) than the light in a place without gravitational field. Then, the light passing near the Sun for example experiments the gravitational redshift when the light is observed at the Earth (less frequency measured, deviation to the red), more wavelength, because of the time clock measure and the real lost energy.

\subsection{Thought Experiment 2}

\section{Inertial Mass=Gravitational Mass}

It is considered the following sequences from the previous thought experiment [1]:

1.- The energy $E$ measured in $A$ is emitted in the form of radiation from A towards $D$, where by the result just obtained, the energy absorbed and measured in $D$ is: $E^{\prime}=E\left(1+\frac{g d}{c^{2}}\right)$

2.- An object of mass $M$ is lowered from A to $D$, doing work $W$ in the process.

3.- The energy $E$ is transferred from $D$ to the object of mass $M$ while it is in $D$. The mass $M$ is now $M{ }^{\prime}$ 'due to this addition of the energy.

4.- The object is raised again to A, doing work in this process.

5.- Let the energy E transferred back from the object to A.

The effect of this cycle is simply that D has experienced the increase in energy $E \frac{g d}{c^{2}}$ of the pulse emitted in A and

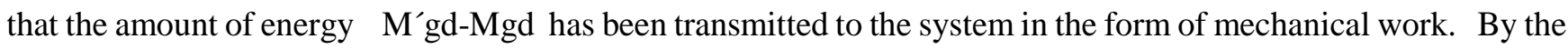
principle of energy, we must have: 
$E \frac{g d}{c^{2}}=\mathrm{M}^{\prime} g d-\mathrm{Mgd}$

$\frac{E}{c^{2}}=\mathrm{M}^{\prime}-\mathrm{M}$

Thus, the increase in gravitational mass is equal to $\mathrm{E} / \mathrm{c}^{2}$ and equal to the increase in inert mass which is established by the theory of relativity. This result is in accordance with the equivalence principle.

\section{Experimental Test}

\subsection{Gravitational Redshift}

The predicted fractional change in frequency from the thought experiment 6.1 is:

$f^{\prime} \cong f\left(1+\frac{g d}{c^{2}}\right) \quad \frac{f^{\prime}-f}{f}=\frac{\Delta f}{f}=\frac{g d}{c^{2}}$

If we consider the distance $\mathrm{d}$ from sea level to the highest mountain on Earth, its value is approximately only $10^{-12}$. In 1960, Pound and Rebka were able to confirm the prediction using the Jefferson Tower at Harvard with a height of $22,5 \mathrm{~m} \mathrm{[5]}$.

$\frac{f^{\prime}-f}{f}=\frac{\Delta f}{f}=\frac{g d}{c^{2}}=\frac{(9,8)(22,5)}{\left(3 * 10^{8}\right)^{2}}=2,45 * 10^{-15}$

Using the Mossbauer effect (which allows a very sensitive measurement of frequency variations) with a gamma ray source and taking great care to control the variables, Pound and Rebka observed this gravitational effect on photons and confirmed the quantitative prediction [5]. Then, Pound and Snider in a subsequent experiment found the same result with more accuracy [5].

The result can be generalized to photons emitted from the surface of stars and observed on Earth. It is assumed that the gravitational field does not need to be uniform and that the result depends only on the potential difference between the source and the observer. The formula for the gravitational redshif was calculated at the thought experiment 6.1:

$f^{\prime} \cong f\left(1-\frac{G M}{R c^{2}}\right)$

It is possible to explain with the next example: $\mathrm{f}$ represents the number of vibrations of an elemental light generator measured by a delicate relay in the same place. This is done on the surface of the Sun where A is located as the thought experiment 6.1. A portion of the light that is emitted from the Sun reaches the Earth (detector D) where we measure the frequency of the light that arrives with a clock $U$ [1]. The measured frequency in $D$ is given by the demonstrated formula:

$f^{\prime} \cong f\left(1-\frac{G M}{R c^{2}}\right) \quad \Phi=\frac{G M}{R}=\mathrm{gd}$

In this case, $M$ is the mass of the Sun and R is the radius of the Sun and $\Phi$ is the gravitational potential of the Sun. Thus, the spectral lines of sunlight must be somewhat redshifted which is the gravitational redshift, compared to the corresponding spectral lines of terrestrial light sources by the approximate amount: 
$\frac{f-f^{\prime}}{f}=\frac{G M}{R c^{2}} \approx 2,11 \times 10^{-6}$

This shift of the spectral lines of sunlight can be measured, but due to other influences (pressure, temperature) that affect the position of the centers of the spectral lines, it is difficult to measure this spectral shift [1].

\subsection{Light Deflection}

Einstein calculated that the light from the stars should be deflected by approximately 1.75 arc seconds when passing close to the sun. Stars that appear near the Sun can only be visible in a total solar eclipse. In 1919, the Royal Society of London organized an expedition to Africa and another to Brazil to observe an eclipse of the sun. The observations showed with great accuracy that the apparent deviation of the position of the stars near the Sun corresponded to that predicted by Einstein [4].

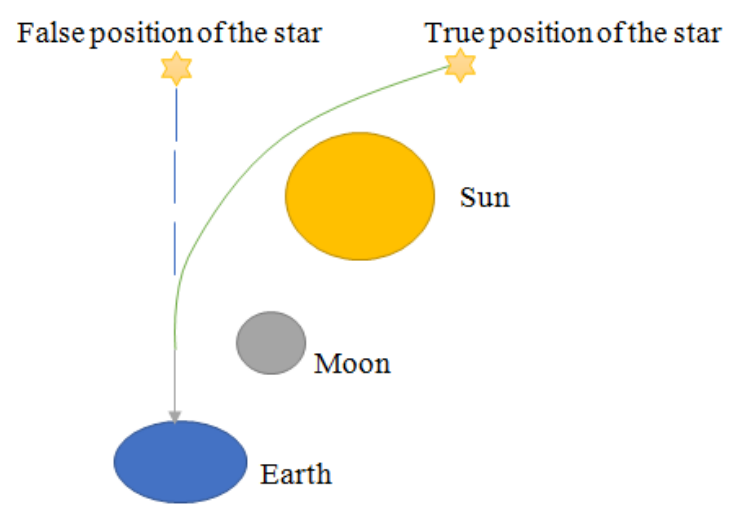

Fig.6 Light Deflection from the stars when passing close to the Sun

\section{Light Deflection by using the velocity formula for the light}

The light velocity is given by the next formula (from 6.1):

$$
\begin{aligned}
& v^{\prime} \cong v_{o}\left(1-2 \frac{G M}{R c^{2}}\right) \\
& d v^{\prime}=2 \frac{v_{o} G M}{c^{2} r^{2}} d r \quad \frac{d v^{\prime}}{d t}=2 \frac{v_{o} G M}{c^{2} r^{2}} \frac{d r}{d t} \quad \frac{d r}{d t}=v^{\prime} \\
& d v^{\prime}=2 \frac{v_{o} G M}{c^{2} r^{2}} v^{\prime} d t \quad \frac{d v^{\prime}}{v^{\prime}}=2 \frac{v_{o} G M}{c^{2} r^{2}} d t \quad d t \cong \frac{d z}{c} \text { (photon time) } \\
& \frac{d v^{\prime}}{v^{\prime}}=2 \frac{v_{o} G M}{c^{2} r^{2}} \frac{d z}{c} \quad \frac{d v^{\prime}}{v^{\prime}}=2 \frac{G M}{c^{2} r^{2}} d z \quad v_{o}=c \\
& d \theta=2 \frac{d v^{\prime} \cos \varphi}{v^{\prime}} \\
& d \theta=2 \frac{G M}{c^{2} r^{2}} d z \cos \varphi=\frac{R}{r} \\
& d \theta=2 \frac{G M}{c^{2} r^{2}} d z \frac{R}{r} \quad d \theta=2 \frac{G M R}{c^{2}} \frac{d z}{\left(R^{2}+z^{2}\right)^{3 / 2}} \quad r=\sqrt{R^{2}+z^{2}} \\
& \theta=2 \frac{G M R}{c^{2}} \int_{-\infty}^{+\infty} \frac{d z}{\left(R^{2}+z^{2}\right)^{3 / 2}}
\end{aligned}
$$


$\mathrm{z}=\operatorname{Rtan}(\vartheta)$

$\theta=2 \frac{G M R}{c^{2}} \frac{1}{R^{3}} \int_{-\pi / 2}^{\pi / 2} \frac{\operatorname{Rec}^{2} \vartheta}{\sec ^{3} \vartheta} d \vartheta$

$\theta \approx \frac{4 G M}{R c^{2}}$

If we replace the values of the mass and radius of the Sun and the light velocity, we obtain: $\theta=1,75$ arc secons of light deflection.
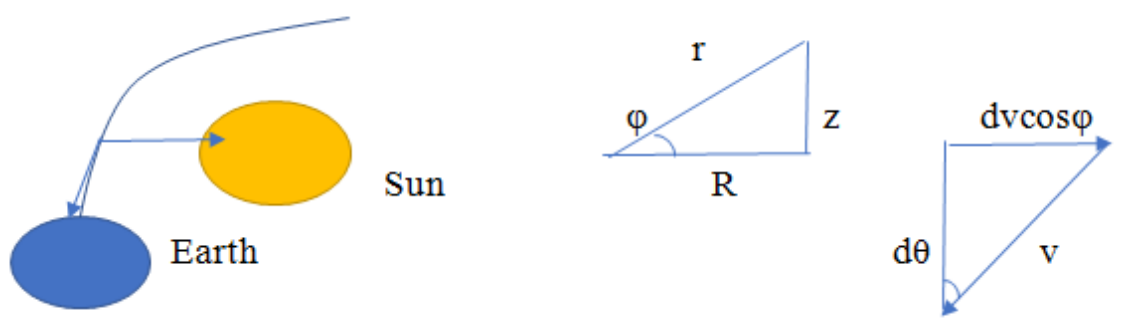

Fig.7 Light deflection with the components of the velocity and the angles $d \theta$ and $\varphi$

\section{Light Deflection by using Relativity Theory}

The four dimensional space time ds in flat space according to spatial relativity is given by the next equation [3], [10]:

$d s^{2}=c^{2} d t^{2}-d l^{2}$

In this case, light follows straight paths which are expressed by the relation:

$d s^{2}=0$

Therefore, we obtain:

$c^{2} d t^{2}-d l^{2}=0 \quad c=\frac{d l}{d t} \quad$ (constant velocity)

But our world is no flat, and then it is necessary to do some corrections due to the curvature of the space time.

Therefore, we consider a spherical distribution of mass $\mathrm{M}$ and the four dimensional space time is given by the next expression [3], [10]:

$d s^{2}=\left(1-\frac{2 G M}{r c^{2}}\right) c^{2} d t^{2}-\left(1+\frac{2 G M}{r c^{2}}\right) d l^{2}$

We can solve the equation $d s^{2}=0$ by using power series [10]:

$0=\left(1-\frac{2 G M}{r c^{2}}\right) c^{2} d t^{2}-\left(1+\frac{2 G M}{r c^{2}}\right) d l^{2}$

$\frac{d l}{c d t}=\frac{\sqrt{\left(1-\frac{2 G M}{r c^{2}}\right)}}{\sqrt{\left(1+\frac{2 G M}{r c^{2}}\right)}} \quad\left(1-\frac{2 G M}{r c^{2}}\right)^{\frac{1}{2}} \cong 1-\frac{G M}{r c^{2}}$ for $\frac{G M}{r} \ll c^{2}$

$\left(1+\frac{2 G M}{r c^{2}}\right)^{-1 / 2} \cong 1-\frac{G M}{r c^{2}}$ for $\frac{G M}{r} \ll c^{2}$ 
$\frac{d l}{c d t} \cong 1-\frac{2 G M}{r c^{2}}$ for $\left(\frac{G M}{r c^{2}}\right)^{2}$ is neglected for $\frac{G M}{r} \ll c^{2}$

$d l=c d t\left(1-\frac{2 G M}{r c^{2}}\right)$

If we divide by dt, we obtain:

$v^{\prime}=v_{o}\left(1-\frac{2 G M}{r c^{2}}\right) \quad$ where $\mathrm{v}_{\mathrm{o}}=\mathrm{c}$

By applying the same procedure of before, it is possible to obtain:

$\theta \approx \frac{4 G M}{R c^{2}} \quad \theta=1,75$ arc secons of light deflection

\subsection{Perihelion Precession of Mercury}

The perihelion or the ellipse of Mercury has a small rotation and changes a small angle $\theta$ after every turn around the Sun [1]. A prediction of the Einstein Theory is that the Perihelion Precession of Mercury must differ of the classic prediction for about 43 arc seconds by century [5].

It is possible to obtain by using the formula demonstrated from 6.1 :

$d^{\prime}=d\left(1-\frac{G M}{a c^{2}}\right) \quad \mathrm{r}=\mathrm{a}$ for an ellipse

where $M$ is the mass of the Sun, $d$ is the perihelion and $c$ is the light velocity: $M=1,989 * 10^{30} \mathrm{~kg}, \mathrm{~d}=45926524600$ $\mathrm{m}, \mathrm{c}=3 * 10^{8} \mathrm{~m} / \mathrm{s}$

a: semi major axis of the ellipse $=57894348600 \mathrm{~m}$

The formula for the period of every Planet is given by Kepler Law [7]:

$T^{2}=\frac{4 \pi^{2}}{G M_{S}} a^{3} \mathrm{~T}=7598971,805 \mathrm{~s}$ for circular motion $\mathrm{a}=\mathrm{r}$ radius of the orbit

$d=r$ : perihelion distance $d^{\prime}$ : the decrease perihelion distance

$\mathrm{d}^{\prime}=45926523431 \mathrm{~m} \quad \Delta \mathrm{d}=\mathrm{d}-\mathrm{d}^{\prime}=1169,352684 \mathrm{~m}$

$\mathrm{a}^{\prime}:$ the decrease semi mayor axis of the ellipse $=\mathrm{a}-\Delta \mathrm{d}=57894347431 \mathrm{~m}$

$T^{\prime 2}=\frac{4 \pi^{2}}{G M_{s}} a^{\prime 3}$

$\mathrm{T}^{\prime}=7598971,575 \mathrm{~s}$

Because of the different time measured in a gravitational field, it is used the formula:

$t=t^{\prime}\left(1-\frac{G M}{R c^{2}}\right) \quad \mathrm{t}^{\prime}=\mathrm{T}^{\prime} \quad \mathrm{t}=\mathrm{T}^{\prime \prime}:$ time in the gravitational field

$\mathrm{T}^{\prime \prime}=7598971,381 \mathrm{~s}$

The perihelion of Mercury has moved an angle $\theta^{\prime}$ : 
$\theta^{\prime}=\frac{2 \pi * T^{\prime \prime}}{T}\left(1-\frac{G M}{a c^{2}}\right) \quad \theta^{\prime}=6,28319949 \mathrm{Rad}$

The factor $\left(1-\frac{G M}{a c^{2}}\right)$ is because the value of $\frac{\text { Perimeter of Circunference }}{\text { radius }}$ in a euclidean geometry is $2 \pi$, but it has an increase value due the decrease of the radius because of the gravitational field.

$\Delta \theta=2 \pi-\theta^{\prime}$

$\Delta \theta=5,1032 * 10^{-7} \mathrm{Rad} / \mathrm{rev}$

1 arcsecond $=4,84 * 10^{-6} \mathrm{Rad}$

$\mathrm{T}_{\text {mer }}=0,241 \mathrm{~T}_{\text {Earth }}$ (relation between periods of Mercury and Earth)

1 century $=100$ years

By applying the factors of arcsecond, $\mathrm{T}_{\text {mer }}$ and century, it is obtained:

$\Delta \theta=43,75$ arcoseconds/century. This value is in agreement with prediction of the value of General Relativity Theory of 43 arcseconds.

The value by using General Relativity Theory is obtained by using the formula [1]:

$\Delta \theta=\frac{24 \pi^{3} a^{2}}{T^{2} c^{2}\left(1-e^{2}\right)}(\operatorname{Rad} / \operatorname{Rev})$ where $\mathrm{e}=0,2056, \mathrm{r}$ is the perihelion of Mercury and $\mathrm{M}_{\mathrm{s}}$ is the mass of the Sun and by applying the factors of before, it is obtained:

$\Delta \theta=43 \operatorname{arcseconds/century}$

\section{Conclusions}

The mass of the particle after the emission of the gravitational energy in a gravitational field (particle orbiting a Planet or Sun) is:

$m=m_{o} \sqrt{\left(1-\frac{v^{2}}{c^{2}}\right)}$ mass for a particle in a gravitational field

$\left.\Delta m=m_{o}\left(1-\sqrt{\left(1-\frac{G M}{r c^{2}}\right.}\right)\right) \quad \Delta \mathrm{m}=\mathrm{m}_{0}-\mathrm{m}: \quad$ total lost mass of the particle

It is in agreement with the total mass decreasing of the particle in a gravitational field for the classic approach:

$\Delta \mathrm{m}=m_{o} c^{2}-m c^{2}=\left(\frac{G M m_{o}}{2 R}\right) \quad \mathrm{GM} / \mathrm{r}<<\mathrm{c}^{2}$

Also, the mass for the particle in a gravitational field which uses a Newton Approach agrees with the formula for the mass from General Relativity at distances very far from the massive object (Sun for example) and also at distances very near to the massive object with high accuracy (4). Then, both models: the Newton Approach with a gravitational field interaction and gravitation force and the Relativity Theory with a curvature of the space time are in agreement at the results for the mass of the particle in a gravitational field with high accuracy. Besides, it is possible to have the next thought experiment: If a mass $M_{o}$ is suspended from a spring balance in the $K$ ' 
accelerated system with acceleration $g$, the balance will indicate the apparent weight $\mathrm{M}_{\circ} \mathrm{g}$ due to the inertia of $\mathrm{M}_{\mathrm{o}}$ (the balance transmits the acceleration to the mass $\mathrm{M}_{\mathrm{o}}$ ) [1].

If the amount of energy $\mathrm{E}$ is transferred to $\mathrm{M}_{\mathrm{o}}$, the spring balance, by the law of the inertia of the energy (equivalence of mass and energy $\mathrm{E} / \mathrm{c}^{2}=\mathrm{m}$ ), will indicate $M_{o} g+\frac{E}{c^{2}} g$. Exactly, this should happen when the experiment is repeated in the gravitational system $\mathrm{K}$ where the balance transmits the gravity acceleration of the Earth to the mass $\mathrm{M}_{\mathrm{o}}$. It happens also at the light deflection when passing close the Sun. The light does not have mass but light has energy and as consequence, it experiments the interaction with the gravitational field by using a Newton Approach [1].

The total energy for a particle in a gravitational field is as follows:

$\mathrm{E}=m c^{2}-m_{o} c^{2}$

$E=m_{o} c^{2}\left(\sqrt{\left(1-\frac{G M}{r c^{2}}\right.}-1\right)$ energy for a particle in a gravitational field

$\mathrm{E}=-\frac{G M m_{0}}{2 r} \quad$ (total energy at the classic approach) $\mathrm{GM} / \mathrm{r}<<\mathrm{c}^{2}$

The total mass energy lost is given by the formula demonstrated and it is equal in absolute value to the total bond energy of the system (particle orbiting a Planet or Sun) which is emitted as gravitational energy when the particle goes from one orbit to another orbit with less radius. The emission of the total gravitational energy E produces a lost mass of the particle $\mathrm{m}=\mathrm{E} / \mathrm{c}^{2}$.

For a Binary Star, the formula for the decrease mass is as follows:

$m_{1}=m_{o 1} \sqrt{\left(1-\frac{v_{1}^{2}}{c^{2}}\right)} \quad G \frac{m_{o 2}^{2}}{r\left(m_{o 1}+m_{o 2}\right)}=v_{1}^{2}$

$m_{2}=m_{o 2} \sqrt{\left(1-\frac{v_{2}^{2}}{c^{2}}\right)} \quad G \frac{m_{o 1}{ }^{2}}{r\left(m_{o 1}+m_{o 2}\right)}=v_{2}^{2}$

Then, each star has different lost mass given by $\Delta \mathrm{m}=\mathrm{m}_{\mathrm{o}} \mathrm{c}^{2}-\mathrm{mc}^{2}$. Also, each star has different gravitational energy emission corresponding to the lost mass of the respective star.

The total energy for each particle is as follows:

$$
\begin{array}{ll}
\mathrm{E}_{1}=\mathrm{m}_{1} \mathrm{c}^{2}-\mathrm{m}_{o 1} \mathrm{c}^{2} m_{1}=m_{o 1} \sqrt{\left(1-\frac{v_{1}{ }^{2}}{c^{2}}\right)} \quad G \frac{m_{o 2}{ }^{2}}{r\left(m_{o 1}+m_{o 2}\right)}=v_{1}{ }^{2} \\
\mathrm{E}_{2}=\mathrm{m}_{2} \mathrm{c}^{2}-\mathrm{m}_{02} \mathrm{c}^{2} m_{2}=m_{o 2} \sqrt{\left(1-\frac{v_{2}{ }^{2}}{c^{2}}\right)} & G \frac{m_{o 1}{ }^{2}}{r\left(m_{o 1}+m_{o 2}\right)}=v_{2}{ }^{2}
\end{array}
$$

Therefore, it is possible to obtain for the total energy at the classic approach:

$$
m_{1}=m_{o 1}\left(1-\frac{v_{1}^{2}}{2 c^{2}}\right) \quad \mathrm{E}_{1}=\mathrm{m}_{1} \mathrm{c}^{2}-\mathrm{m}_{\mathrm{o} 1} \mathrm{c}^{2}=-\frac{v_{1}{ }^{2}}{2} m_{o 1}
$$


$m_{2}=m_{o 2}\left(1-\frac{v_{2}^{2}}{2 c^{2}}\right) \quad \mathrm{E}_{2}=\mathrm{m}_{2} \mathrm{c}^{2}-\mathrm{m}_{\mathrm{o} 2} \mathrm{c}^{2}=-\frac{v_{2}^{2}}{2} m_{o 2}$

The total energy of the system is:

$E=-G \frac{m_{o 1} m o_{2}}{2 r}$ as the classic result

For the light, the formula obtained for the gravitational redshift frequency, wavelength, time measurement, decrease distance and light velocity are:

$f^{\prime} \cong f\left(1-\frac{G M}{R c^{2}}\right)$ gravitational redshift for the light

Where, $\mathrm{M}$ is the mass of the planet or Sun and $\mathrm{R}$ is the respective radius.

It is possible to obtain this formula by using the variant mass due the gravitational Potential Energy:

$m=m_{o} e^{\frac{-G M}{c^{2}}\left(\frac{1}{R}\right)}$ (Variant Mass due the Gravitational Potential Energy)

By replacing for the light $\mathrm{mc}^{2}=\mathrm{hf}$ and $\mathrm{GM} / \mathrm{r}<<\mathrm{c}^{2}$, it is obtained:

$f^{\prime} \cong f\left(1-\frac{G M}{R c^{2}}\right) \quad$ gravitational redshift for the light

The wavelength is given by the next formula:

$\lambda^{\prime}=\lambda\left(1-\frac{G M}{R c^{2}}\right) \quad \lambda=\lambda_{\mathrm{G}}:$ increased wavelength

The formula for the time in a gravitational field is:

$t^{\prime}=t /\left(1-\frac{G M}{R c^{2}}\right) \quad \mathrm{t}:$ clock time with the gravitational field

Also, the formula for the decrease distance $\mathrm{d}$ of a particle orbiting a Planet due the gravitational energy emission is as follows:

$d^{\prime}=d\left(1-\frac{G M}{r c^{2}}\right) \quad$ decrease distance due the gravitational energy emission

The light velocity $\mathrm{v}^{\prime}=\lambda^{\prime} \mathrm{f}^{\prime}$ is obtained by replacing the respective formulas $\lambda^{\prime}$ and $\mathrm{f}^{\prime}$ or $v^{\prime}=\frac{d^{\prime}}{t^{\prime}}$ :

$v^{\prime} \cong v_{o}\left(1-2 \frac{G M}{R c^{2}}\right)$ light velocity in a gravitational field $\Phi$

In resume, in a gravitational field, the light has lower velocity, lower rates of the clock-time (less time measured) than the light in a place without gravitational field. Then, the light passing near the Sun for example experiments the gravitational redshift when the light is observed a the Earth (less frequency measured, deviation to the red), more wavelength, because of the time clock measured and the real lost energy.

By using the formula for the light velocity, the value of the light deflection is:

$\theta \approx \frac{4 G M}{R c^{2}} \quad \theta=1,75$ arc secons of light deflection. It is in agreement with the result of the General Relativity Theory. Also, the formula for time dilation and decrease distance are used to calculate the Perihelion Precession of 
Mercury. The result $\Delta \theta=43,75$ arcoseconds/century is in agreement with the experiment result and with the Relativity Theory with high accuracy.

This research is relevant for a unification theory. Photons and particles are used indistinctly at this research (for example the formula of the gravitational redshift frequency for the light is obtained from the formula of the variant mass due the gravitational potential). Besides, it is used the energy $\mathrm{E}=\mathrm{hf}$ for the photon energy at the demonstration of the gravitational redshift formula (at the thought experiment 6.1).

It can be concluded from this research that gravitational and electromagnetic energy are radiation of the same kind but with different frequency in the spectrum electromagnetic. This research has profound relation of the results for quantum mechanics as for example with the Atom of Bohr and it constitutes an important step for a unification theory. The same process occurs in the atom for the electron when jump from one orbit. The particle decreases its radio and the velocity $\mathrm{v}$ increases with emission of electromagnetic energy (photon) at the jump of the electron from one orbit to another with less radius. Besides, the electron losses mass as result of this emission. It is established at the article Variant Mass for an Accelerated Charged Particle [2]. It is possible to appreciate the symmetry of the physics and the nature by comparing what happen at the atom (microscopic scale) and at the planetary system or space (macroscopic scale).

\section{References}

[1] Stephen Hawking, La Gran Ilusión, Las Grandes Obras de Albert Einstein, Editorial Crítica, Barcelona, España, 2009.

[2] Giovanni Alcocer, Variant Mass for an accelerated charged particle, Mediterranean Journal of Basic and Applied Sciences, MJBAS, August 2020.

[3] Tolga Yarman, Alexander Kholmetskii, Metin Arik, Bending of light caused by gravitation: the same result via totally different philosophies, Okan University, Istanbul, Turkey.

[4] Manuel Torres, Física Moderna, Editorial Limusa, México, 1981.

[5] Robert Resnick, Introducción a la Teoría Especial de la Relatividad, Editorial Limusa, México, 1981.

[6] Wilson, Física Quinta Edición, Prentice Hall, México, 2003.

[7] Serway, Física, Mc Graw Hill, México, 1993.

[8] Robert C. Hilborn, Gravitational waves from orbiting binaries without general relativity: a tutorial, American Association of Physics Teachers, 2019.

[9] Real Sociedad Española de Física R.S.E.F., El Experimento de Cavendish, Universidad de Alicante.

[10] Peter Coles, Einstein, Eddington and the 1919 Eclipse, School of Physics and Astronomy, University of Nottingham. 